\title{
Gentisic Acid, Salicylic Acid, Total Phenolic Content and Cholinesterase Inhibitory Activities of Red Wines Made from Various Grape Varieties
}

\author{
A. Stój ${ }^{1 *}$, D. Szwajgier ${ }^{1}$, E. Baranowska-Wójcik ${ }^{1}$, D. Domagała ${ }^{2}$
}

(1) Department of Biotechnology, Microbiology and Human Nutrition, Faculty of Food Science and Biotechnology, University of Life Sciences, Skromna 8, 20-704 Lublin, Poland

(2) Department of Applied Mathematics and Computer Science, Faculty of Production Engineering, University of Life Sciences, Głęboka 28, 20-612 Lublin, Poland

Submitted for publication: April 2018

Accepted for publication: July 2018

Key words: Gentisic acid, salicylic acid, phenolics, acetylcholinesterase activity, butyrylcholinesterase activity, red wine

\begin{abstract}
Alzheimer's disease is characterised by a decrease in acetylcholine (ACh) levels in the brain due to the activity of acetylcholinesterase ( $\mathrm{AChE}$ ) and butyrylcholinesterase (BChE). As a result, disorders in cholinergic transmission are observed, leading to cognitive impairment. In this work, the inhibition of $\mathrm{AChE}$ and $\mathrm{BChE}$ activities by red wines made of various grape varieties was determined for the first time. There was no significant difference in the polyphenol content between the grape varieties; nevertheless, there were significant differences in the content of gentisic acid and salicylic acid, and in the inhibition of $\mathrm{AChE}$ and $\mathrm{BChE}$ between the wine samples. A statistically significant correlation between AChE inhibitory activity and salicylic acid, as well as between $\mathrm{BChE}$ inhibitory activity and total phenolic content, was recorded. In model solution studies, it was shown that salicylic acid effectively inhibited BChE activity at concentrations similar to the maximum concentrations found in the test wines. Hierarchical cluster analysis (HCA) revealed that the wines could be divided into three groups. Cabernet Sauvignon and Syrah wines had the highest content of salicylic acid and $\mathrm{AChE}$ inhibitory activity, as well as low $\mathrm{BChE}$ inhibitory activity. Pinot noir, Tempranillo, Regent and Rondo wines showed the lowest content of salicylic acid and low AChE inhibitory activity. Garnacha tinta, Merlot, Montepulciano and Negroamaro wines had a medium content of salicylic acid, and the highest or medium BChE inhibitory activity. This work is important for both the wine industry and for health protection.
\end{abstract}

\section{INTRODUCTION}

Alzheimer's disease (AD) is an age-related neurodegenerative disease associated with the progressive impairment of cognition. The characteristic features include the accumulation of amyloid beta $(A \beta)$ plaques and neurofibrillary tangles (NFT) in brain tissues (Anand et al., 2017; Kumar et al., 2018). Studies using murine models have shown that dietary polyphenols from wines and other grape products reduce the clinical symptoms of AD (Wang et al., 2006, 2010). Another important aspect related to $\mathrm{AD}$ is the decrease in acetylcholine ( $\mathrm{ACh}$ ) levels, an essential neurotransmitter playing a major role in learning and memory (Anand et al., 2017; Kumar et al., 2018). $\mathrm{ACh}$ is hydrolysed by acetylcholinesterase (AChE) and butyrylcholinesterase (BChE), leading to disorders in the cholinergic transmission. AChE and BChE inhibitors, widely used for the medical treatment of $\mathrm{AD}$, exert negative sideeffects, like gastrointestinal disturbances, nausea, vomiting and diarrhoea, and thus the quest for novel $\mathrm{AChE}$ and $\mathrm{BChE}$ inhibitors is a priority (Gironés-Vilaplana et al., 2012; Erdem et al., 2016). Phenolic compounds naturally occurring in food have received much attention as alternative agents for therapy in AD. Anticholinesterase activity has previously been shown in coffee (Erdem et al., 2016), tea (Okello et al., 2012), fruits (Borowiec et al., 2014), vegetables (Nwanna et al., 2016) and juices (Gironés-Vilaplana et al., 2012), but until now, wines have not been pointed out as possible sources of cholinesterase inhibitors.

As indicated by epidemiological studies in elderly patients, light to moderate wine consumption (particularly a dry, red wine) can be neuroprotective, although heavy alcohol consumption is neurotoxic (Kim et al., 2012). Moderate wine consumption, rather than alcohol consumption per se, has been related to a lower risk of AD (Nooyens et al., 2014). Wines contain polyphenols, a factor that distinguishes them 
from most other alcoholic beverages (Stockley, 2015). Wine polyphenols classified as flavonoids include anthocyanins, flavan-3-ols, flavonols, flavones, isoflavones, flavanones and non-flavonoids, including hydroxybenzoic acids, cinnamic acids and stilbenes (Cotea et al., 2012; Fanzone et al., 2012; Sen \& Tokatli, 2014; Ren et al., 2017; Wojdyło et al., 2018). The most common hydroxybenzoic acids found in wines are gallic acid, gentisic acid, $p$-hydroxybenzoic acid, protocatechuic acid, syringic acid, salicylic acid and vanillic acid (Gonçalves et al., 2013). Szwajgier (2013a) studied the anticholinesterase activity of hydroxybenzoic acids and cinnamic acids using model solutions of phenolic acids. Among the hydroxybenzoic acids found in wines, the highest anticholinesterase activity was exhibited by gentisic and salicylic acids. The aim of this study was to determine the content of both phenolic acids in wines, followed by an examination of the anticholinesterase activities of these phenolic acids at concentrations usually found in a number of wines present in the Polish market.

Previously, gentisic acid and salicylic acid were determined in food samples, including wines, using a UV-Vis detector (Nikfardjam et al., 1999; Xi et al., 2010) or DAD detector (Pereira et al., 2010; Cotea et al., 2012; Fanzone et al., 2012; Ma et al., 2014). In some studies, gentisic acid was detected, but salicylic acid was not (Del Alamo Sanza et al., 2004; Pereira et al., 2010; Fanzone et al., 2012; Gonçalves et al., 2013) and vice versa (e.g. Xi et al., 2010). In a considerable number of publications concerning wines, the determination of gentisic acid and salicylic acid has been omitted altogether (Sen \& Tokatli, 2014; Van Leeuw et al., 2014; Ivanova-Petropulos et al., 2015; Socha et al., 2015; Radovanovic et al., 2016; Tomaz \& Maslov, 2016; Kapusta et al., 2018).

In our work, spectrofluorometric detection was used for the first time to determine the gentisic and salicylic acids in red wines. In the past, authors used a fluorometric detector for the analysis of phenolic compounds in wines (e.g. Vitrac et al., 2002; Tomaz \& Maslov, 2016), but none of these works detected gentisic and salicylic acids. Spectrofluorometric detection has many advantages over UV-Vis detection (higher sensitivity and selectivity due to the unique use of specific excitation and emission wavelengths) (GarmónLobato et al., 2004).

The aim of this work was to determine the content of gentisic acid and salicylic acid, total polyphenol content and, as a novelty, AChE and BChE inhibitory activities in 36 wines produced using ten grape varieties. Furthermore, the inhibition of $\mathrm{AChE}$ and $\mathrm{BChE}$ by gentisic and salicylic acids was examined in model solutions containing both phenolic acids (singly and in the mixture), at maximal concentrations of both compounds detected in the studied wines. This work is important for the wine industry, because it shows the content of gentisic and salicylic acids in red wines made from different grape varieties, and presents the effects of these phenolic acids on the inhibition of cholinesterases, which play an important role in Alzheimer's disease.

\section{MATERIALS AND METHODS \\ Wine samples}

Thirty-six commercial red wines were studied, produced from various varieties of Vitis vinifera: Cabernet Sauvignon (four samples), Garnacha tinta (two samples), Merlot (four samples), Montepulciano (three samples), Negroamaro (three samples), Pinot noir (two samples), Regent (four samples), Rondo (five samples), Syrah (three samples) and Tempranillo (six samples). The grapes were harvested in 2008, 2009 and 2010. The wines originated from different regions of the following countries: France, Italy, Spain and Poland. All wines were bottled at the place of production. French, Italian and Spanish wines had denominations of origin or were regional wines. In Poland, there were no indications of the wine quality of the 2008, 2009 and 2010 vintages. Two bottles of $750 \mathrm{ml}$ each were used for wine sampling. The basic characteristics of the wines are presented in Table 1.

TABLE 1

Description of red wine samples

\begin{tabular}{|c|c|c|c|c|c|}
\hline Wine sample code & Grape variety & Country & Production area & Vintage & Quality \\
\hline CS 1 & Cabernet Sauvignon & France & Languedoc & 2008 & Vin de Pays \\
\hline CS 2 & Cabernet Sauvignon & France & Languedoc & 2010 & Vin de Pays \\
\hline CS 3 & Cabernet Sauvignon & Italy & Trentino & 2008 & DOC \\
\hline $\mathrm{CS} 4$ & Cabernet Sauvignon & Italy & Veneto & 2009 & IGT \\
\hline Gr 1 & Garnacha tinta & Spain & Aragon & 2009 & DO \\
\hline Gr 2 & Garnacha tinta & Spain & Navarra & 2010 & $\mathrm{DO}$ \\
\hline Mr 1 & Merlot & France & Languedoc & 2009 & Vin de Pays \\
\hline $\operatorname{Mr} 2$ & Merlot & France & Bordeaux & 2009 & $\mathrm{AOC}$ \\
\hline Mr 3 & Merlot & France & Bordeaux & 2010 & $\mathrm{AOC}$ \\
\hline $\operatorname{Mr} 4$ & Merlot & Italy & Veneto & 2009 & IGT \\
\hline
\end{tabular}


TABLE 1 (CONTINUED)

\begin{tabular}{|c|c|c|c|c|c|}
\hline Wine sample code & Grape variety & Country & Production area & Vintage & Quality \\
\hline Mp 1 & Montepulciano & Italy & Abruzzo & 2010 & DOC \\
\hline $\operatorname{Mp} 2$ & Montepulciano & Italy & Abruzzo & 2009 & DOC \\
\hline Mp 3 & Montepulciano & Italy & Marche & 2010 & DOC \\
\hline $\mathrm{Ng} 1$ & Negroamaro & Italy & Apulia & 2008 & IGT \\
\hline $\mathrm{Ng} 2$ & Negroamaro & Italy & Apulia & 2010 & DOC \\
\hline $\mathrm{Ng} 3$ & Negroamaro & Italy & Apulia & 2009 & IGT \\
\hline PN 1 & Pinot noir & France & Loire Valley & 2008 & Vin de Pays \\
\hline PN 2 & Pinot noir & Poland & Lower Silesia & 2008 & \\
\hline $\operatorname{Rg} 1$ & Regent & Poland & Lower Silesia & 2008 & \\
\hline $\operatorname{Rg} 2$ & Regent & Poland & Podkarpackie Province & 2008 & \\
\hline $\operatorname{Rg} 3$ & Regent & Poland & Malopolskie Province & 2009 & \\
\hline $\operatorname{Rg} 4$ & Regent & Poland & Lublin Province & 2010 & \\
\hline $\operatorname{Rd} 1$ & Rondo & Poland & Mazowieckie Province & 2010 & \\
\hline $\operatorname{Rd} 2$ & Rondo & Poland & Mazowieckie Province & 2008 & \\
\hline $\operatorname{Rd} 3$ & Rondo & Poland & Lubuskie Province & 2009 & \\
\hline $\operatorname{Rd} 4$ & Rondo & Poland & Lublin Province & 2010 & \\
\hline $\operatorname{Rd} 5$ & Rondo & Poland & Lublin Province & 2010 & \\
\hline Sh 1 & Syrah & France & Languedoc & 2009 & Vin de Pays \\
\hline Sh 2 & Syrah & France & Rhone Valley & 2008 & $\mathrm{AOC}$ \\
\hline Sh 3 & Syrah & Italy & Sicily & 2010 & IGT \\
\hline Tp 1 & Tempranillo & Spain & Castilla-Leon & 2009 & DO \\
\hline $\operatorname{Tp} 2$ & Tempranillo & Spain & Castilla-La Mancha & 2008 & Vino de la Tierra \\
\hline $\operatorname{Tp} 3$ & Tempranillo & Spain & Castilla-La Mancha & 2008 & Vino de la Tierra \\
\hline $\operatorname{Tp} 4$ & Tempranillo & Spain & Rioja & 2010 & DO \\
\hline $\operatorname{Tp} 5$ & Tempranillo & Spain & Castilla-La Mancha & 2010 & DO \\
\hline Tp 6 & Tempranillo & Spain & Castilla-La Mancha & 2010 & DO \\
\hline
\end{tabular}

\section{Chemicals}

Gentisic and salicylic acids (HPLC standards) were at $98 \%$ and $\geq 99 \%$ purity respectively. AChE (from Electrophorus electricus, C3389), BChE (from equine serum, C7512), acetylthiocholineiodide(ATCh, 01480), S-butyrylthiocholine chloride (BTCh, B3128), 5,5'-dithiobis-2-nitrobenzoic acid (DTNB, D8130), eserine (E8375), Folin-Ciocalteu reagent, dimethyl sulfoxide (DMSO), formic acid, quercetin, Tris$\mathrm{HCl}$ buffer, ethanol (of analytical grade) and methanol (for HPLC, Chromasolv, $\geq 99.9 \%$, 34860) were purchased from Sigma-Aldrich, Poznań. Sodium carbonate was from P.O.Ch. (Lublin, Poland).

\section{HPLC of phenolic acids}

The Knauer HPLC system consisted of two Smartline 100 pumps, a dynamic mixer and a $20 \mu 1$ loop. Detection was performed using a Merck Hitachi Fluorescence Detector (coupled with a Knauer IF2 interface), with maximum excitation/emission at $325 \mathrm{~nm} / 440 \mathrm{~nm}$. A Macherey-Nagel LiChospher $100 \mathrm{RP} 18(250 \mathrm{~mm} \times 4.0 \mathrm{~mm}, 5 \mu \mathrm{m})$ column, coupled with a corresponding pre-column, was used for separations. The flow was $1 \mathrm{ml} / \mathrm{min}$, with the working pressure not exceeding $28 \mathrm{MPa}$. The optimised gradient was formed by deionised water containing $0.1 \%$ formic acid (A) and $70 \%$ methanol in deionised water with $0.1 \%$ formic acid 
(B): 0 to $5 \min 100 \% \mathrm{~A}, 5$ to $60 \min 0$ to $100 \% \mathrm{~B}, 60$ to 63 min $100 \%$ B, 63 to $66 \min 100$ to $0 \% \mathrm{~B}, 66$ to $70 \min 100 \%$ A. Signals were analysed using Eurochrom 3.05 P5 software. Wine samples were filtered $(0.45 \mu \mathrm{m}$, Millipore $)$ and directly injected in triplicate. Stock solutions of both phenolic acids ( $1 \mathrm{~g} / \mathrm{L}$ each) were prepared by dissolving the HPLC standards in methanol, followed by dilution with deionised water to obtain final solutions (in the range 0.05 to $2.5 \mathrm{mg} / \mathrm{L}$ for gentisic acid and 0.26 to $4.17 \mathrm{mg} / \mathrm{L}$ for salicylic acid) and injected into the HPLC-FLD system. Concentrations against peak area were plotted. LOD and LOQ were defined as the concentrations for which signal-to-noise ratios $(\mathrm{S} / \mathrm{N})$ were 3 and 10 respectively. The intra-day repeatability and interday reproducibility were determined by the injection of six individual replicates of standard solutions within one day and on three different days respectively. Reproducibility was expressed as relative standard deviation (RSD) for the peak area.

\section{Determination of total phenolic content (TPC)}

The TPC of the wines was determined using Folin-Ciocalteu (F-C) reagent according to Clarke et al. (2013). Ten $\mu \mathrm{L}$ of a wine diluted appropriately in dimethyl sulfoxide (DMSO) was mixed with $100 \mu \mathrm{L}$ of F-C reagent freshly diluted with distilled water (1:10). After $5 \mathrm{~min}$, the solution was mixed with $100 \mu \mathrm{L}$ of $7.5 \%(\mathrm{w} / \mathrm{v})$ sodium carbonate solution. The sample was then left for $60 \mathrm{~min}$, before measurement of the absorbance at $650 \mathrm{~nm}$ against a blank (DMSO) in a 96well microplate absorbance reader (Tecan Sunrise, Grödig, Austria). Four replicate measurements were performed.

TABLE 2

Validation data for phenolic acids obtained by HPLC-FLD

\begin{tabular}{lccccccc}
\hline & $\begin{array}{c}\text { Concentration } \\
\text { range }(\mathrm{mg} / \mathrm{L})\end{array}$ & Calibration curve & $\mathrm{R}^{2}$ & $\begin{array}{c}\text { LOD } \\
(\mathrm{mg} / \mathrm{L})\end{array}$ & $\begin{array}{c}\text { LOQ } \\
(\mathrm{mg} / \mathrm{L})\end{array}$ & $\begin{array}{c}\text { Repeatability } \\
(\mathrm{RSD}, \%)\end{array}$ & $\begin{array}{c}\text { Reproducibility } \\
(\mathrm{RSD}, \%)\end{array}$ \\
\hline Gentisic acid & $0.05-2.5$ & $\mathrm{y}=1.4365 \mathrm{x}-0.1052$ & 0.997 & 0.015 & 0.049 & 0.22 & 0.29 \\
Salicylic acid & $0.26-4.17$ & $\mathrm{y}=1.633 \mathrm{x}-0.2307$ & 0.998 & 0.031 & 0.102 & 0.14 & 0.68 \\
\hline
\end{tabular}
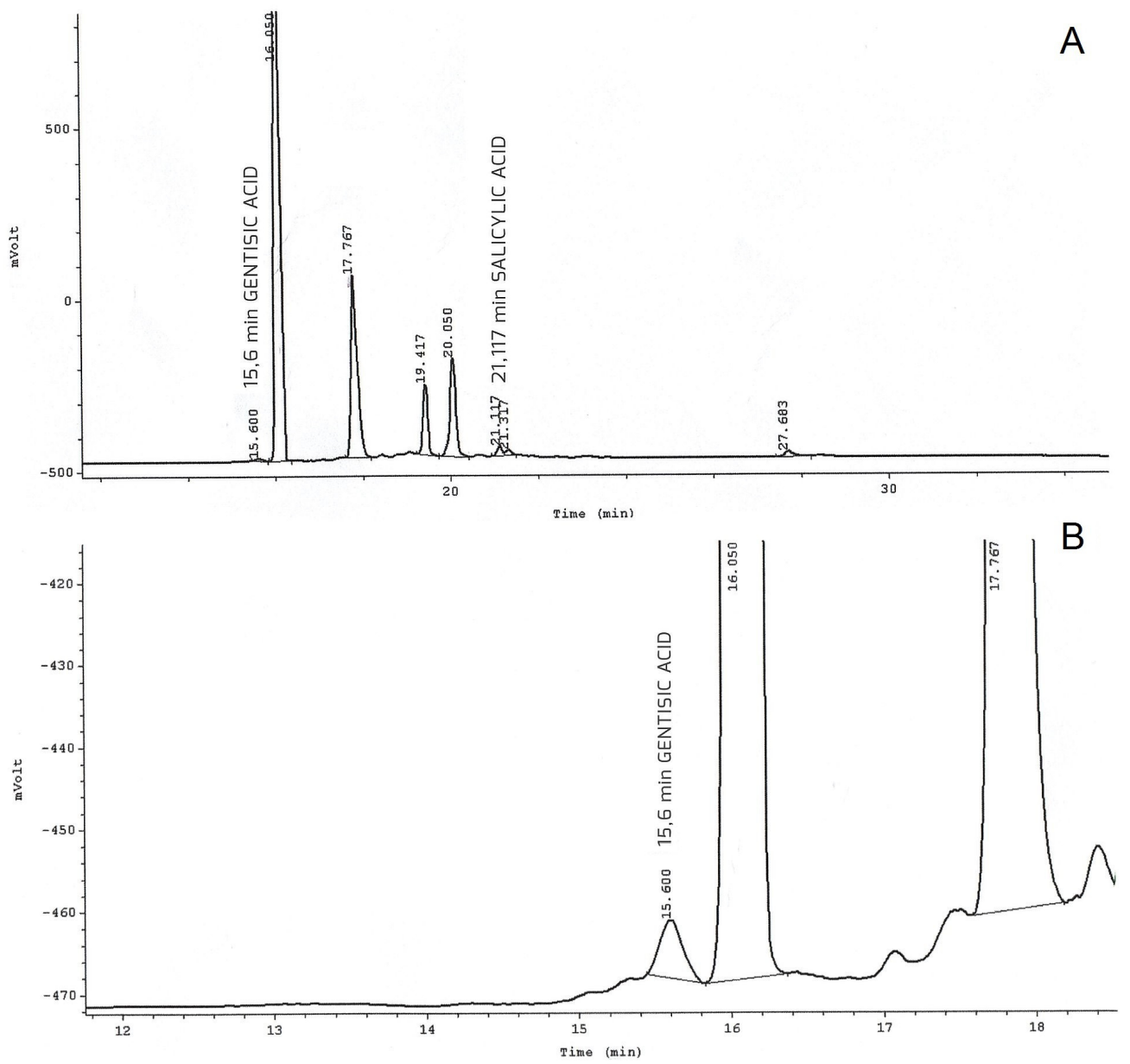

FIGURE 1

HPLC-FLD chromatogram of Rondo wine sample (Rd 4). A - whole chromatogram, B - a magnification showing a peak of gentisic acid (see Table 1 for wine sample code). 
TABLE 3

Contents of gentisic acid, salicylic acid, total phenolic compounds (TPC), AChE and BChE inhibitory activities in red wines produced from different grape varieties.

\begin{tabular}{|c|c|c|c|c|c|}
\hline $\begin{array}{l}\text { Wine } \\
\text { sample } \\
\text { code }\end{array}$ & $\begin{array}{c}\text { Gentisic acid } \pm \text { SD } \\
\mathrm{mg} / \mathrm{L}\end{array}$ & $\begin{array}{c}\text { Salicylic acid } \pm \text { SD } \\
\mathrm{mg} / \mathrm{L}\end{array}$ & $\begin{array}{c}\mathrm{TPC} \pm \mathrm{SD} \\
\mathrm{mg} / \mathrm{L}\end{array}$ & $\begin{array}{c}\mathrm{AChE} \pm \mathrm{SD} \\
\mu \mathrm{mol} / \mathrm{L}\end{array}$ & $\begin{array}{c}\mathrm{BChE} \pm \mathrm{SD} \\
\mu \mathrm{mol} / \mathrm{L}\end{array}$ \\
\hline CS 1 & $1.35 a \pm 0.05$ & $9.44 a b \pm 0.13$ & $2004.70 \mathrm{ab} \pm 36.53$ & $0.339 a \pm 0.02$ & $0.200 \mathrm{ab} \pm 0.02$ \\
\hline CS 2 & $0.37 \mathrm{a} \pm 0.01$ & $12.79 a \pm 0.12$ & $2498.62 a \pm 126.80$ & $0.223 \mathrm{ab} \pm 0.01$ & $0.183 b \pm 0.01$ \\
\hline CS 3 & $1.33 a \pm 0.06$ & $10.69 \mathrm{ab} \pm 0.16$ & $1893.08 \mathrm{ab} \pm 55.53$ & $0.352 \mathrm{a} \pm 0.03$ & $0.280 \mathrm{a} \pm 0.02$ \\
\hline $\mathrm{CS} 4$ & $0.58 a \pm 0.02$ & $7.66 \mathrm{~b} \pm 0.21$ & $1475.20 b \pm 65.54$ & $0.205 b \pm 0.01$ & $0.237 \mathrm{ab} \pm 0.01$ \\
\hline Gr 1 & $<\mathrm{LOQ}$ & $4.25 \mathrm{a} \pm 0.03$ & $2361.89 a \pm 95.88$ & $0.100 \mathrm{a} \pm 0.01$ & $0.603 a \pm 0.02$ \\
\hline Gr 2 & n.d. & $5.51 b \pm 0.11$ & $1956.57 b \pm 77.55$ & $0.073 b \pm 0.01$ & $0.814 b \pm 0.03$ \\
\hline Mr 1 & $0.20 \mathrm{a} \pm 0.02$ & $5.62 \mathrm{ab} \pm 0.08$ & $2064.70 \mathrm{ab} \pm 71.67$ & $0.105 \mathrm{ab} \pm 0.01$ & $0.696 a \pm 0.07$ \\
\hline Mr 2 & $0.31 \mathrm{a} \pm 0.01$ & $11.34 \mathrm{a} \pm 0.19$ & $2944.41 \mathrm{a} \pm 49.78$ & $0.049 \mathrm{ab} \pm 0.01$ & $0.206 b \pm 0.02$ \\
\hline Mr 3 & $0.20 \mathrm{a} \pm 0.01$ & $6.53 \mathrm{ab} \pm 0.20$ & $2608.85 \mathrm{ab} \pm 37.20$ & $0.022 \mathrm{a} \pm 0.00$ & $0.222 \mathrm{ab} \pm 0.02$ \\
\hline $\operatorname{Mr} 4$ & $0.28 \mathrm{a} \pm 0.01$ & $4.02 b \pm 0.04$ & $1814.25 b \pm 70.76$ & $0.166 b \pm 0.02$ & $0.258 \mathrm{ab} \pm 0.01$ \\
\hline Mp 1 & n.d. & $8.78 a \pm 0.90$ & $1865.88 \mathrm{ab} \pm 44.84$ & $0.311 \mathrm{a} \pm 0.05$ & $0.183 a \pm 0.01$ \\
\hline Mp 2 & $0.15 a \pm 0.01$ & $6.12 \mathrm{a} \pm 0.14$ & $1820.53 a \pm 30.57$ & $0.213 b \pm 0.01$ & $0.272 \mathrm{ab} \pm 0.02$ \\
\hline Mp 3 & $<\mathrm{LOQ}$ & $7.94 a \pm 0.16$ & $2338.87 b \pm 30.77$ & $0.276 \mathrm{ab} \pm 0.02$ & $0.471 b \pm 0.02$ \\
\hline $\mathrm{Ng} 1$ & $0.33 \mathrm{ab} \pm 0.01$ & $6.82 \mathrm{a} \pm 0.27$ & $2546.76 \mathrm{ab} \pm 92.76$ & $0.275 \mathrm{ab} \pm 0.02$ & $0.308 \mathrm{a} \pm 0.01$ \\
\hline $\mathrm{Ng} 2$ & $0.25 b \pm 0.01$ & $7.41 \mathrm{a} \pm 0.13$ & $1636.36 b \pm 28.55$ & $0.283 b \pm 0.02$ & $0.209 \mathrm{a} \pm 0.01$ \\
\hline $\mathrm{Ng} 3$ & $0.75 a \pm 0.03$ & $6.57 \mathrm{a} \pm 0.10$ & $3268.81 \mathrm{a} \pm 48.23$ & $0.180 \mathrm{a} \pm 0.01$ & $0.211 \mathrm{a} \pm 0.01$ \\
\hline PN 1 & $0.10 \mathrm{a} \pm 0.01$ & n.d. & $1940.52 \mathrm{a} \pm 61.14$ & $0.155 \mathrm{a} \pm 0.02$ & $0.047 \mathrm{a} \pm 0.01$ \\
\hline PN 2 & $0.11 \mathrm{a} \pm 0.01$ & $6.10 \mathrm{a} \pm 0.09$ & $2076.56 b \pm 31.60$ & $0.022 b \pm 0.00$ & $0.397 b \pm 0.02$ \\
\hline $\operatorname{Rg} 1$ & $2.81 \mathrm{a} \pm 0.08$ & $6.90 \mathrm{a} \pm 0.10$ & $2511.18 \mathrm{ab} \pm 116.86$ & $0.154 \mathrm{a} \pm 0.016$ & $0.400 \mathrm{ab} \pm 0.03$ \\
\hline $\operatorname{Rg} 2$ & n.d. & $0.45 b \pm 0.02$ & $1468.9 \mathrm{a} 3 \mathrm{~b} \pm 53.97$ & $0.060 \mathrm{ab} \pm 0.01$ & $0.091 \mathrm{ab} \pm 0.01$ \\
\hline $\operatorname{Rg} 3$ & $2.29 \mathrm{ab} \pm 0.05$ & $<\mathrm{LOQ}$ & $3176.02 \mathrm{a} \pm 27.00$ & $0.098 \mathrm{ab} \pm 0.01$ & $1.067 \mathrm{a} \pm 0.02$ \\
\hline $\operatorname{Rg} 4$ & $0.97 b \pm 0.03$ & $<\mathrm{LOQ}$ & $1166.85 b \pm 51.83$ & $0.021 b \pm 0.00$ & $0.053 b \pm 0.00$ \\
\hline $\mathrm{Rd} 1$ & $0.22 \mathrm{a} \pm 0.01$ & $0.52 \mathrm{a} \pm 0.02$ & $3178.81 \mathrm{a} \pm 55.31$ & $0.195 \mathrm{a} \pm 0.01$ & $0.545 \mathrm{a} \pm 0.02$ \\
\hline $\operatorname{Rd} 2$ & $0.23 \mathrm{ab} \pm 0.01$ & n.d. & $1910.52 \mathrm{ab} \pm 70.83$ & $0.022 b \pm 0.00$ & $0.047 b \pm 0.00$ \\
\hline $\operatorname{Rd} 3$ & n.d. & n.d. & $1385.91 b \pm 65.38$ & $0.055 \mathrm{ab} \pm 0.00$ & $0.304 \mathrm{ab} \pm 0.01$ \\
\hline $\operatorname{Rd} 4$ & $0.86 b \pm 0.03$ & $3.376 b \pm 0.06$ & $1037.09 b \pm 38.08$ & $0.021 b \pm 0.00$ & $0.054 b \pm 0.00$ \\
\hline $\operatorname{Rd} 5$ & n.d. & n.d. & $2483.9 \pm 101.70$ & $0.072 \mathrm{ab} \pm 0.01$ & $0.412 \mathrm{a} \pm 0.01$ \\
\hline Sh 1 & n.d. & $11.86 \mathrm{a} \pm 0.26$ & $1791.93 \mathrm{ab} \pm 49.63$ & $0.344 \mathrm{ab} \pm 0.03$ & $0.047 \mathrm{a} \pm 0.00$ \\
\hline Sh 2 & $0.18 \mathrm{a} \pm 0.01$ & $11.09 \mathrm{ab} \pm 0.38$ & $1712.40 \mathrm{a} \pm 34.43$ & $0.125 a \pm 0.00$ & $0.061 \mathrm{ab} \pm 0.00$ \\
\hline Sh 3 & $<\mathrm{LOQ}$ & $4.74 b \pm 0.15$ & $1990.75 b \pm 75.74$ & $0.352 b \pm 0.02$ & $0.331 b \pm 0.02$ \\
\hline
\end{tabular}




\begin{tabular}{lccccc}
$\begin{array}{l}\text { Wine } \\
\text { sample } \\
\text { code }\end{array}$ & $\begin{array}{c}\text { Gentisic acid } \pm \mathrm{SD} \\
\mathrm{mg} / \mathrm{L}\end{array}$ & $\begin{array}{c}\text { Salicylic acid } \pm \mathrm{SD} \\
\mathrm{mg} / \mathrm{L}\end{array}$ & $\begin{array}{c}\text { TPC } \pm \mathrm{SD} \\
\mathrm{mg} / \mathrm{L}\end{array}$ & $\begin{array}{c}\text { AChE } \pm \mathrm{SD} \\
\mu \mathrm{mol} / \mathrm{L}\end{array}$ & $\begin{array}{c}\text { BChE } \pm \mathrm{SD} \\
\mu \mathrm{mol} / \mathrm{L}\end{array}$ \\
\hline Tp 1 & $<\mathrm{LOQ}$ & $3.48 \mathrm{ab} \pm 0.07$ & $2569.78 \mathrm{a} \pm 77.86$ & $0.171 \mathrm{a} \pm 0.01$ & $0.866 \mathrm{a} \pm 0.03$ \\
Tp 2 & $0.15 \mathrm{ab} \pm 0.01$ & $1.76 \mathrm{ab} \pm 0.06$ & $2142.83 \mathrm{ab} \pm 104.92$ & $0.023 \mathrm{~b} \pm 0.00$ & $0.181 \mathrm{~b} \pm 0.01$ \\
Tp 3 & $0.10 \mathrm{a} \pm 0.01$ & $4.30 \mathrm{a} \pm 0.24$ & $1860.29 \mathrm{~b} \pm 85.42$ & $0.025 \mathrm{~b} \pm 0.00$ & $0.118 \mathrm{~b} \pm 0.01$ \\
Tp 4 & $<$ LOQ & $1.36 \mathrm{ab} \pm 0.05$ & $2234.92 \mathrm{ab} \pm 25.42$ & $0.105 \mathrm{ab} \pm 0.00$ & $0.951 \mathrm{a} \pm 0.03$ \\
Tp 5 & $0.28 \mathrm{ab} \pm 0.01$ & $0.75 \mathrm{~b} \pm 0.03$ & $1877.74 \mathrm{~b} \pm 81.72$ & $0.086 \mathrm{ab} \pm 0.01$ & $0.275 \mathrm{ab} \pm 0.01$ \\
Tp 6 & $0.92 \mathrm{~b} \pm 0.02$ & $<$ LOQ & $2292.82 \mathrm{ab} \pm 49.47$ & $0.110 \mathrm{ab} \pm 0.01$ & $0.584 \mathrm{ab} \pm 0.03$ \\
\hline
\end{tabular}

Different letters in the same column for the same grape variety indicate significantly different means $(\mathrm{p}<0.05)$ among wines from selected grape varieties

TABLE 4

Mean contents of gentisic acid, salicylic acid, total phenolics (TPC), AChE and BChE inhibitory activities in red wines produced from different grape varieties

\begin{tabular}{|c|c|c|c|c|c|}
\hline Grape variety & $\begin{array}{c}\text { Gentisic acid } \\
\mathrm{mg} / \mathrm{L}\end{array}$ & $\begin{array}{c}\text { Salicylic acid } \\
\mathrm{mg} / \mathrm{L}\end{array}$ & $\begin{array}{l}\mathrm{TPC} \\
\mathrm{mg} / \mathrm{L}\end{array}$ & $\begin{array}{l}\mathrm{AChE} \\
\mu \mathrm{mol} / \mathrm{L}\end{array}$ & $\begin{array}{r}\mathrm{BChE} \\
\mu \mathrm{mol} / \mathrm{L}\end{array}$ \\
\hline Cabernet Sauvignon & $0.91 \mathrm{a} \pm 0.51$ & $10.14 a \pm 2.16$ & $1967.90 \mathrm{a} \pm 383.0$ & $0.280 \mathrm{a} \pm 0.07$ & $0.225 b c \pm 0.04$ \\
\hline Garnacha tinta & $0.02 c \pm 0.03$ & $4.88 \mathrm{abcd} \pm 0.89$ & $2159.23 a \pm 231.2$ & $0.087 b c \pm 0.02$ & $0.708 \mathrm{a} \pm 0.12$ \\
\hline Merlot & $0.25 \mathrm{abc} \pm 0.05$ & $6.88 \mathrm{abc} \pm 3.15$ & $2358.05 a \pm 461.6$ & $0.086 c \pm 0.06$ & $0.345 \mathrm{abc} \pm 0.21$ \\
\hline Montepulciano & $0.06 \mathrm{c} \pm 0.07$ & $7.61 \mathrm{abc} \pm 1.27$ & $2008.42 \mathrm{a} \pm 247.0$ & $0.267 \mathrm{a} \pm 0.05$ & $0.308 \mathrm{abc} \pm 0.13$ \\
\hline Negroamaro & $0.44 \mathrm{ab} \pm 0.27$ & $6.93 \mathrm{abc} \pm 0.43$ & $2483.97 \mathrm{a} \pm 699.9$ & $0.246 \mathrm{ab} \pm 0.05$ & $0.243 b c \pm 0.05$ \\
\hline Pinot noir & $0.11 \mathrm{abc} \pm 0.01$ & $3.05 \mathrm{bcd} \pm 4.31$ & $2008.54 \mathrm{a} \pm 85.5$ & $0.089 b c \pm 0.07$ & $0.222 b c \pm 0.19$ \\
\hline Regent & $1.52 \mathrm{a} \pm 1.27$ & $1.87 \mathrm{~cd} \pm 3.30$ & $2080.7 a \pm 834.2$ & $0.083 c \pm 0.05$ & $0.403 \mathrm{abc} \pm 0.42$ \\
\hline Rondo & $0.26 \mathrm{abc} \pm 0.35$ & $0.78 \mathrm{~d} \pm 1.47$ & $1999.26 a \pm 788.6$ & $0.073 c \pm 0.07$ & $0.272 b c \pm 0.20$ \\
\hline Syrah & $0.07 b c \pm 0.09$ & $9.23 \mathrm{ab} \pm 3.91$ & $1831.69 a \pm 132.3$ & $0.274 \mathrm{a} \pm 0.11$ & $0.146 c \pm 0.14$ \\
\hline Tempranillo & $0.25 \mathrm{abc} \pm 0.34$ & $1.95 \mathrm{~cd} \pm 1.60$ & $2163.07 \mathrm{a} \pm 259.3$ & $0.087 \mathrm{c} \pm 0.05$ & $0.496 \mathrm{ab} \pm 0.34$ \\
\hline
\end{tabular}

Different letters in the same column for the same grape variety indicate significantly different means $(\mathrm{p}<0.05)$ among wines from selected grape varieties. Missing data were replaced with substituted values using multiple imputation.

The TPC was expressed in quercetin equivalents, based on a calibration curve prepared the same way as the studied samples, using quercetin at 0 to $500 \mathrm{mg} / \mathrm{L}$.

\section{Inhibition of cholinesterases}

Inhibition of ChEs by wine samples, model solutions of gentisic and salicylic acids, and mixtures of both phenolic acids, was evaluated using the modified method of Ellman et al. (1961). Solutions of reagents prepared daily were dissolved in Tris- $\mathrm{HCl}$ buffer (100 mmol, $\mathrm{pH}$ 8.0). The reaction mixture was composed of: $0.035 \mathrm{~mL}$ of the studied

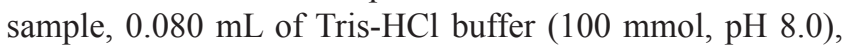
$0.035 \mathrm{~mL}$ of ATCh or BTCh (1.5 mmol), $0.175 \mathrm{~mL}$ of DTNB (0.3 mmol DTNB containing $10 \mathrm{mmol} \mathrm{NaCl}$ and $2 \mathrm{mmol}$ $\mathrm{MgCl}_{2} \cdot 6 \mathrm{H}_{2} \mathrm{O}$ ), and $0.020 \mathrm{~mL}$ of $\mathrm{AChE}$ or $\mathrm{BChE}$ solution $(0.28$ units $/ \mathrm{mL})$. The absorbance $\left(405 \mathrm{~nm}, 22^{\circ} \mathrm{C}\right)$ was read after $25 \mathrm{~min}(\mathrm{BChE})$ or $18 \mathrm{~min}(\mathrm{AChE})$ using a 96well microplate absorbance reader (Tecan Sunrise, Grödig,
Austria). Blank samples containing either eserine (181 $\mu \mathrm{mol})$ or Tris- $\mathrm{HCl}$ buffer instead of a studied sample were examined simultaneously and called a positive and negative control respectively. A blank sample containing DTNB and ATCh (or BTCh) and adjusted to the final reaction volume with Tris- $\mathrm{HCl}$ buffer was used to control the spontaneous hydrolysis of the substrate. In addition, a so-called falsepositive (f-p) sample was run according to the method of Rhee et al. (2003). The f-p sample consisted of $0.035 \mathrm{~mL}$ of ATCh or BTCh, $0.020 \mathrm{~mL}$ of AChE or BChE solution, and $0.080 \mathrm{~mL}$ of Tris-HCl buffer incubated for $25 \mathrm{~min}$ (BChE) or $18 \mathrm{~min}$ (AChE). After the incubation, $0.035 \mathrm{~mL}$ of wine sample and $0.175 \mathrm{~mL}$ of DTNB were added and the absorbance was read immediately, as described above. If tiocholine released in the f-p sample during the incubation combined with compounds from the wine sample, the resulting lower tiocholine concentration would result in a decreased absorbance compared with the negative sample. 
TABLE 5

Contents of gentisic acid, salicylic acid and total phenolic compounds (TPC) in red wines produced from different grape varieties determined by other authors.

\begin{tabular}{|c|c|c|c|}
\hline Compounds & Grape variety & Concentration $\mathrm{mg} / \mathrm{L}$ & References \\
\hline Gentisic acid & Cabernet Sauvignon & 9.93011 & Cotea et al. (2012) \\
\hline Gentisic acid & Cabernet Sauvignon & 2.03 & Ma et al. (2014) \\
\hline Gentisic acid & Cabernet Sauvignon & 0.93 & Fanzone et al. (2012) \\
\hline Gentisic acid & Merlot & 1.91 & Ma et al. (2014) \\
\hline Gentisic acid & Tempranillo & $2.745-6.323$ & Del Alamo Sanza et al. (2004) \\
\hline Salicylic acid & Cabernet Sauvignon & 11.37 & Ma et al. (2014) \\
\hline Salicylic acid & Cabernet Sauvignon & $5.30-7.94$ & Xi et al. (2010) \\
\hline Salicylic acid & Cabernet Sauvignon & 4.54604 & Cotea et al. (2012) \\
\hline Salicylic acid & Cabernet Sauvignon & $0.43 \mathrm{mg} / \mathrm{L}$ & Nikfardjam et al. (1999) \\
\hline Salicylic acid & Pinot noir & n.d. -0.31 & Nikfardjam et al. (1999) \\
\hline Salicylic acid & Merlot & 15.26 & Ma et al. (2014) \\
\hline Salicylic acid & Negroamaro & 0.116 & Barnaba et al. (2016) \\
\hline $\mathrm{TPC}$ & Cabernet Sauvignon & $1787-2413$ & Raczkowska et al. (2011) \\
\hline $\mathrm{TPC}$ & Cabernet Sauvignon & $1791-2131$ & Ivanova-Petropulos et al. (2015) \\
\hline $\mathrm{TPC}$ & Cabernet Sauvignon & $2284-3355$ & Sen and Tokatli (2014) \\
\hline $\mathrm{TPC}$ & Cabernet Sauvignon & $1673-3503$ & Ginjom et al. (2010) \\
\hline $\mathrm{TPC}$ & Cabernet Sauvignon & $2029-2880$ & Van Leeuw et al. (2014) \\
\hline $\mathrm{TPC}$ & Cabernet Sauvignon & 2631 & Ma et al. (2014) \\
\hline TPC & Cabernet Sauvignon & $2765.3-3944.3$ & Fanzone et al. (2012) \\
\hline $\mathrm{TPC}$ & Merlot & $1886-3610$ & Ginjom et al. (2010) \\
\hline $\mathrm{TPC}$ & Merlot & $1687-1867$ & Raczkowska et al. (2011) \\
\hline $\mathrm{TPC}$ & Merlot & $1646-3364$ & Sen and Tokatli (2014) \\
\hline TPC & Merlot & $1940-2962$ & Van Leeuw et al. (2014) \\
\hline TPC & Merlot & $1918-2564$ & Ivanova-Petropulos et al. (2015) \\
\hline TPC & Syrah & $1650-2510$ & Ginjom et al. (2010) \\
\hline $\mathrm{TPC}$ & Syrah & $1962-3150$ & Sen and Tokatli (2014) \\
\hline TPC & Syrah & $1763-3476$ & Van Leeuw et al. (2014) \\
\hline TPC & Syrah & $1612-1991$ & Ivanova-Petropulos et al. (2015) \\
\hline $\mathrm{TPC}$ & Pinot noir & $948-3267$ & Van Leeuw et al. (2014) \\
\hline $\mathrm{TPC}$ & Rondo & 1668.57 & Socha et al. (2015) \\
\hline $\mathrm{TPC}$ & Regent & 996.17 & Socha et al. (2015) \\
\hline
\end{tabular}

The samples were analysed in eight replicates.

Model solutions of phenolic acids were prepared by dissolving each compound in ethanol, followed by dilution with deionised water to obtain $3 \mathrm{mg} / \mathrm{L}$ (gentisic acid) and 12 $\mathrm{mg} / \mathrm{L}$ (salicylic acid). Also, a mixture of both phenolic acids was prepared to examine whether there was any synergism or antagonism between the compounds. The concentration of ethanol in test solutions of phenolic acids was similar to that measured in wine samples (typically $13 \% \mathrm{v} / \mathrm{v}$ ). This ethanol content in a sample has no influence on the cholinesterase activity, as was previously estimated (data not published); ethanol up to $20 \%(\mathrm{v} / \mathrm{v})$ in a final sample had no effect on the enzymatic activity.

The inhibitory activity of wines was expressed in eserine equivalents, as previously proposed (Szwajgier \& Borowiec, 2012a; Szwajgier, 2013a), with the use of calibration curves of eserine (in the range 0.007 to $0.12 \mu \mathrm{mol} / \mathrm{L}$ for $\mathrm{AChE}$ and 0.013 to $0.35 \mu \mathrm{mol} / \mathrm{L}$ for $\mathrm{BChE})$. 


\section{Statistical analysis}

The Statistica 13.0 software package (StatSoft, Krakow, Poland) was used for data analysis. The nonparametric Kruskal-Wallis test was used to verify the presence of probable statistically significant differences between wines from different grape varieties, as well as within each variety. In the cases in which statistically significant differences were found, post hoc tests were performed at the $p<0.05$ significance level. Spearman's correlation coefficients were calculated in order to determine the strength of the relationship between the tested compounds for all the wines. Hierarchical cluster analysis (HCA) was used in order to separate the studied wine samples into groups (Ward method, with a similarity matrix based on the Euclidean distance) (Romesburg, 2004; Ma et al., 2014)

\section{RESULTS AND DISCUSSION}

Previously, authors have prepared wine samples before injection into the HPLC system (liquid-liquid extraction, e.g. Xi et al., 2010; Ma et al., 2014; Socha et al., 2015; solidphase extraction, e.g. Fanzone et al., 2012; Barnaba et al., 2016; Tomaz \& Maslov, 2016; microextraction by packed sorbent, e.g. Gonçalves et al., 2013). Other workers have injected wine samples without any preparation step (Pereira et al., 2010; Raczkowska et al., 2011; Sen \& Tokatli, 2014; Van Leeuw et al., 2014). A complicated, multi-step sample preparation may lead to the modification of phenolic composition and to the loss of phenolics (Vitrac et al., 2002). It was therefore decided to omit the sample preparation procedure by using spectrofluorometric detection, which is highly selective.

Table 2 shows regression equations, regression coefficients $\left(\mathrm{R}^{2}\right)$, limits of detection (LOD) and limits of quantification (LOQ), intra-day repeatability and inter-day reproducibility for gentisic and salicylic acid. The method resulted in a linear response over the studied range of concentrations, low values of LOD and LOQ, as well as good repeatability and reproducibility. The LOD and LOQ results are lower than limits obtained by other authors, who used HPLC-DAD or UHPLC-DAD (Pereira et al., 2010; Gonçalves et al., 2013). However, Cotea et al. (2012) achieved lower levels for LOD and LOQ in comparison to our work. Fig. 1 shows an example of a HPLC-FLD chromatogram of wine produced in Poland from the Rondo variety.

The contents of gentisic and salicylic acids, TPC, AChE and $\mathrm{BChE}$ inhibitory activities in 36 red wines produced from 10 grape varieties are shown in Table 3, while mean values calculated for each variety are presented in Table 4. Results are discussed for the individual parameters below.

\section{Gentisic acid content of red wines}

In a number of the wines examined, gentisic acid was not detected (Garnacha tinta wine: Gr 2, Montepulciano wine: Mp 1, Regent wine: $\operatorname{Rg} 2$, Rondo wines: $\operatorname{Rd} 3$ and $\operatorname{Rd} 5$, Syrah wine: Sh 1) (Table 3). The content of gentisic acid, salicylic acid and TPC determined by other authors are presented in Table 5.

The highest gentisic acid concentration was detected in Regent wine $(\operatorname{Rg} 1 ; 2.81 \mathrm{mg} / \mathrm{L})$. Taking grape varieties into consideration, statistically significant differences $(p<0.05)$ were observed in the content of gentisic acid within selected varieties (Negroamaro, Regent, Rondo, Tempranillo), although the wines originated from the same countries. On the other hand, no significant differences $(p>0.05)$ were found in wines produced from the Pinot noir variety, although they originated from different countries. Also, there were no statistically significant differences $(p>0.05)$ in Cabernet Sauvignon and Merlot wines, regardless of whether they originated from the same country or from different countries.

When comparing grape varieties, the mean content of gentisic acid in wines produced from the Cabernet Sauvignon and Regent varieties was significantly higher $(\mathrm{p}<0.05)$ in comparison with wines produced from the Garnacha tinta and Montepulciano varieties (Table 4). Furthermore, the mean content of gentisic acid in wines produced from the Cabernet Sauvignon variety was higher than in those produced from the Merlot variety, which is in agreement with the results obtained by Ma et al. (2014).

In this study, the concentration of gentisic acid in Cabernet Sauvignon wines varied from $0.37 \mathrm{mg} / \mathrm{L}$ to 1.35 $\mathrm{mg} / \mathrm{L}$, and the average content was $0.91 \mathrm{mg} / \mathrm{L}$. Cotea et al. (2012) and Ma et al. (2014) detected a higher concentration of gentisic acid in Cabernet Sauvignon wines (Table 5). In the study of Fanzone et al. (2012), Cabernet Sauvignon wines contained gentisic acid in a similar range, and Merlot concentrations ranged from $0.20 \mathrm{mg} / \mathrm{L}$ to $0.31 \mathrm{mg} / \mathrm{L}$ (average concentration of $0.25 \mathrm{mg} / \mathrm{L})$. Ma et al. (2014) reported higher concentrations of this acid in Merlot wines. Gentisic acid was found in Tempranillo wines in our study, at concentrations ranging from < LOQ to $0.92 \mathrm{mg} / \mathrm{L}$ and at an average concentration of $0.25 \mathrm{mg} / \mathrm{L}$, whereas Del Alamo Sanza et al. (2004) detected gentisic acid at higher concentrations.

\section{Salicylic acid content of red wines}

Salicylic acid was either not detected in the selected wines (Pinot noir: PN 1, Rondo: Rd 2, Rd 3 and Rd 5), or reached a maximum of $12.79 \mathrm{mg} / \mathrm{L}$ in Cabernet Sauvignon wine (CS 2) (Table 3$)$. Statistically significant differences $(\mathrm{p}<0.05)$ in the content of salicylic acid were found within selected grape varieties, regardless of whether the wines originated from the same country or from different countries, except for the Montepulciano and Negroamaro varieties.

When grape varieties were compared, Cabernet Sauvignon wines showed a significantly higher mean salicylic acid content compared to Rondo wines (Table 4). Furthermore, the mean content of salicylic acid in Cabernet Sauvignon wines was higher than in Merlot, which is consistent with the results of Jin et al. (2017).

Salicylic acid was present in the Cabernet Sauvignon wines examined in our work at concentrations, ranging from $7.66 \mathrm{mg} / \mathrm{L}$ to $12.79 \mathrm{mg} / \mathrm{L}$ (average content of $10.14 \mathrm{mg} / \mathrm{L}$ ), whereas other authors reported similar, lower or higher contents of salicylic acid in their wines produced from the same grape variety (Table 5). In this study, salicylic acid was detected in wines produced from the Pinot noir variety at a maximum concentration of $6.10 \mathrm{mg} / \mathrm{L}$ (average content $3.05 \mathrm{mg} / \mathrm{L}$ ), while Nikfardjam et al. (1999) found this compound at lower concentrations. Our study indicates that wines produced from the Merlot variety contained $4.02 \mathrm{mg}$ 
to $11.34 \mathrm{mg}$ salicylic acid/L, with an average content of $6.88 \mathrm{mg} / \mathrm{L}$. According to Ma et al. (2014), salicylic acid was present at higher concentrations in Merlot wine. The highest discrepancies in the content of salicylic acid were found in Negroamaro wines, where levels from $6.57 \mathrm{mg} / \mathrm{L}$ to $7.41 \mathrm{mg} / \mathrm{L}$ were determined, whereas Barnaba et al. (2016) determined a concentration of only $0.116 \mathrm{mg} / \mathrm{L}$ in their study.

\section{Total phenolic content (TPC) of red wines}

The range of TPC found in the red wines in this study was from $1037.09 \mathrm{mg} / \mathrm{L}$ to $3268.81 \mathrm{mg} / \mathrm{L}$. The lowest TPC was recorded for Rondo wine ( $\mathrm{Rd} 4)$, while Negroamaro wine (Ng 3) was characterised by the highest TPC value (Table 3). Within each grape variety, there were statistically significant differences $(\mathrm{p}<0.05)$ between samples.

When comparing grape varieties, no differences $(p>0.05)$ between mean values of TPC were found (Table 4), which is in agreement with Ginjom et al. (2010). The highest TPC values were observed in wines produced from the Negroamaro variety (2 $483.97 \mathrm{mg} / \mathrm{L}$ ), and the lowest TPC values were observed in wines produced from the Syrah variety (1 $831.69 \mathrm{mg} / \mathrm{L})$. A comparison of mean TPC of wines produced from selected varieties indicated that Merlot wines had higher TPC means than Cabernet Sauvignon wines, which in turn had higher value than Syrah wines. These data are consistent with results presented by Ginjom et al. (2010), Sen and Tokatli (2014) and IvanovaPetropulos et al. (2015). However, in the paper of Van Leeuw et al. (2014), TPC decreased in the order Syrah > Cabernet Sauvignon > Merlot, and in the paper of Ma et al. (2014), TPC levels in Cabernet Sauvignon wines were higher in comparison to those in Merlot wines.

In our study, TPC in Cabernet Sauvignon wines ranged from $1475.20 \mathrm{mg} / \mathrm{L}$ to $2498.62 \mathrm{mg} / \mathrm{L}$, and the average content
TABLE 6

Spearman's correlation coefficients between parameters of wines.

\begin{tabular}{lcccc}
\hline & BChe & TPC & $\begin{array}{c}\text { Gentisic } \\
\text { acid }\end{array}$ & $\begin{array}{c}\text { Salicylic } \\
\text { acid }\end{array}$ \\
\hline AChE & 0.104 & 0.075 & 0.037 & $\mathbf{0 . 5 5 2}$ \\
BChE & & $\mathbf{0 . 5 3 1}$ & -0.063 & -0.152 \\
TPC & & 0.169 & 0.066 \\
Gentisic acid & & & & 0.168 \\
\hline
\end{tabular}

Bold indicates statistical significance at $\mathrm{p}<0.05$

was $1967.90 \mathrm{mg} / \mathrm{L}$. Other authors have reported similar or higher concentrations of TPC in Cabernet Sauvignon wines (Table 5). Our results concerning TPC in wines produced from the Merlot, Syrah, Pinot noir and Rondo varieties are comparable with those presented elsewhere (Ginjom et al., 2010; Raczkowska et al., 2011; Sen \& Tokatli, 2014; Van Leeuw et al., 2014; Ivanova-Petropulos et al., 2015; Socha et al., 2015). However, TPC levels detected in Regent in our study were higher than those reported by Socha et al. (2015).

\section{Cholinesterase inhibitory activities of red wines}

The inhibition of AChE and BChE has not been studied in wines so far. Szwajgier and Borowiec (2012b) screened fruits and vegetables for cholinesterase inhibitors and reported that grape extract exhibited similar inhibition of AChE and BChE $(0.98 \mu \mathrm{mol}$ eserine/L). A comparison of inhibitory activities relating to $\mathrm{AChE}$ and $\mathrm{BChE}$ revealed that the BChE inhibitory activity was higher than the AChE inhibitory activity in the case of seven grape varieties out of the 10 varieties studied.

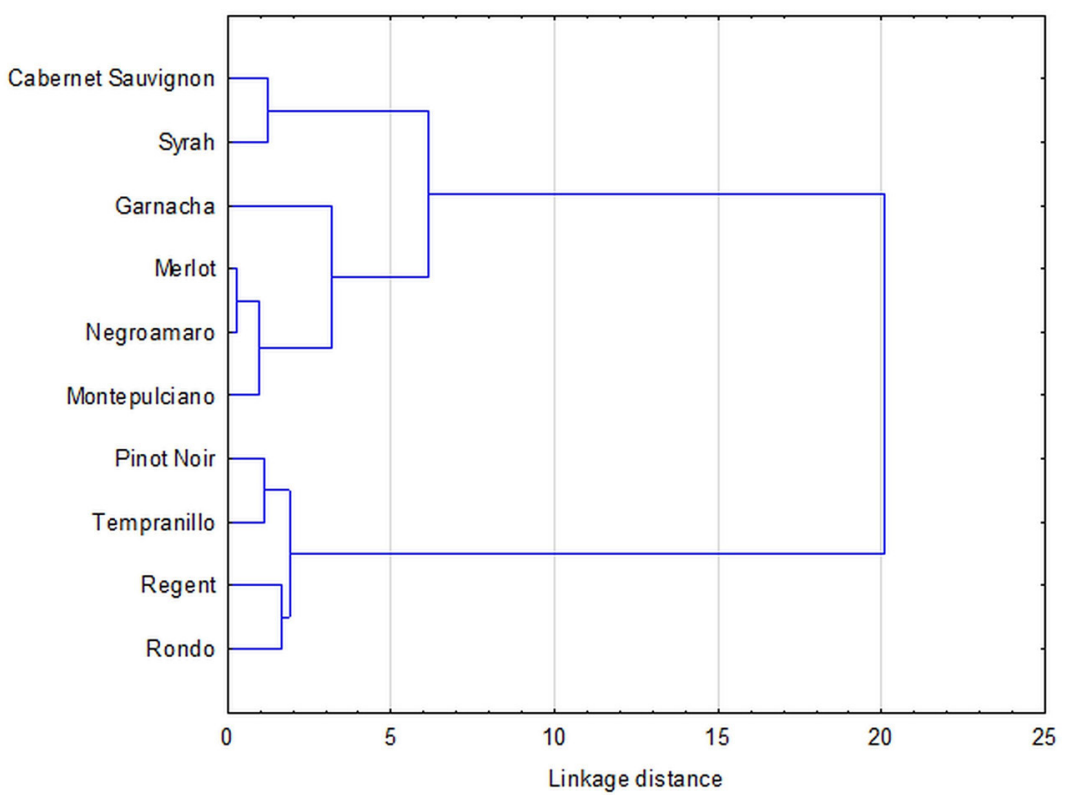

FIGURE 2

Dendrogram presenting the similarity between the studied wines in relation to the content of gentisic acid and salicylic acid, and $\mathrm{AChE}$ and $\mathrm{BChE}$ inhibitory activities 
The AChE inhibitory activity in this study was in the range of $0.021 \mu \mathrm{mol}$ eserine/ $\mathrm{L}$ in wines produced from the Regent ( $\operatorname{Rg} 4$ ) and Rondo ( $\mathrm{Rd} 4$ ) varieties to $0.352 \mu \mathrm{mol}$ eserine/L in wines produced from Cabernet Sauvignon (CS 3) and Syrah (Sh 3). Within each grape variety, statistically significant differences $(p<0.05)$ were observed in the inhibition of $\mathrm{AChE}$, regardless of whether the wines originated from the same country or a variety of countries (Table 3). Between varieties, the mean AChE inhibitory activity of Cabernet Sauvignon, Montepulciano and Syrah wines was significantly higher $(\mathrm{p}<0.05)$ than that detected in Merlot, Regent, Rondo and Tempranillo wines (Table 4).

The BChE inhibitory activity ranged between 0.047 $\mu \mathrm{mol} / \mathrm{L}$ in Pinot noir (PN 1), Rondo (Rd 2) and Syrah (Sh 1) wines and $1.067 \mu \mathrm{mol} / \mathrm{L}$ in Regent ( $\operatorname{Rg} 3$ ) wine. The differences in $\mathrm{BChE}$ inhibitory activity within grape varieties were statistically significant $(\mathrm{p}<0.05)$ in most wines, except Negroamaro wine (Table 3 ). When comparing varieties, the wines produced from the Garnacha tinta variety indicated a statistically higher $(p<0.05)$ mean value of the inhibitory activity in comparison with wines produced from the Syrah variety (Table 4).

\section{Cholinesterase inhibitory activities of gentisic and salicylic acids in model solutions}

The examination of the content of gentisic and salicylic acids in wines revealed that their maximum content values reached $2.82 \mathrm{mg} / \mathrm{L}$ and $12.79 \mathrm{mg} / \mathrm{L}$ respectively. The inhibition of $\mathrm{AChE}$ and $\mathrm{BChE}$ by gentisic and salicylic acids in model solutions was examined at concentrations similar to the maximum content of the acids in wine samples $(3 \mathrm{mg} / \mathrm{L}$ and $12 \mathrm{mg} / \mathrm{L}$ respectively). Also, model solution containing a mixture of those two phenolic acids was studied in the same way. Gentisic acid was inefficient in inhibiting the AChE or $\mathrm{BChE}$ at concentrations found in wines in our work. Also, salicylic acid was inefficient as an AChE inhibitor, but this phenolic acid inhibited BChE activity at $0.063 \mu \mathrm{mol}$ eserine/L. Furthermore, mixtures of both phenolic acids were not able to inhibit $\mathrm{AChE}$, but $\mathrm{BChE}$ was inhibited at $0.041 \mu \mathrm{mol}$ eserine/L. This indicates that there is no synergy between the two phenolic acids, at least at concentrations at which both phenolic acids were detected in the wines studied. According to Szwajgier (2013b), a synergistic and/ or antagonistic effect of two phenolic acids may depend on the site of action of the compound in the cholinesterase, which is determined by hydrophilic and/or hydrophobic binding of the ligand to the enzyme. If a phenolic acid is located in the peripheral site of the enzyme, it can block the entrance of a substrate at the narrow aromatic gorge of the enzyme. Therefore, it can be speculated that the inhibition of cholinesterases detected in wines in our study is caused by the presence of other polyphenols (and/or other components).

\section{Correlation}

A correlation analysis was performed between the examined quality indicators (gentisic acid, salicylic acid, TPC, AChE and BChE) measured in the studied wines. The Spearman's correlation coefficients are presented in Table 6.

The parameters are very weakly correlated with one another. Statistically significant correlation occurred between AChE inhibitory activity and salicylic acid (0.552), and between BChE inhibitory activity and phenolics (0.531). Nwanna et al. (2016) calculated Pearson correlation coefficients between the ChE inhibitory effects of vegetable extracts and some phenolic compounds (gallic acid, catechin, caffeic acid, epicatechin, ellagic acid, orientin, rutin, quercitrin, isoquercitrin, quercetin, kaempferol and chlorogenic acid). These authors noted that caffeic acid had the highest correlation with the AChE inhibitory effect of the extracts (0.449), and gallic and ellagic acid had the highest correlations with the BChE inhibitory effect (0.374, the same value for both acids). Also, Szwajgier and Borowiec (2012a) found a correlation among the total phenolic contents in malts at different mashing stages vs. AChE and BChE inhibitory activities.

\section{Hierarchical cluster analysis}

Taking into account the content of gentisic acid, salicylic acid, and $\mathrm{AChE}$ and $\mathrm{BChE}$ inhibitory activities (no significant differences, $\mathrm{p}<0.05$, in the TPC), and applying hierarchical cluster analysis (HCA) to our dataset, the dendrogram reported in Fig. 2 was obtained.

Reading the dendrogram from left to right, wines that are most similar to each other can be identified. At a similarity level of 4 , three groups of wines are observed. The first group includes the Cabernet Sauvignon and Syrah wines that were characterised by the highest content of salicylic acid and AChE inhibitory activity as well as low BChE inhibitory activity. Wines from the second group - Pinot noir, Tempranillo, Regent and Rondo - were characterised by the lowest content of salicylic acid and low AChE inhibitory activity. The third group includes Garnacha tinta, Merlot, Montepulciano and Negroamaro wines, which contain salicylic acid at an intermediate level. The third group is further divided into two subgroups, one including Merlot, Montepulciano and Negroamaro wines, which have BChE inhibitory activity on a medium level, and the other (only Garnacha tinta) characterised by the highest BChE inhibitory activity. Similarly, Ma et al. (2014) applied HCA with the use of phenolic components as variables for the successful clustering of red wines made of different grape varieties grown in China. A cluster was formed by Cabernet Sauvignon and Merlot wines, another by Cabernet Gernischt and Cinsault wines, and a third cluster by Gamay wines. In turn, Radovanovic et al. (2016) observed shorter clustering distances in the case of Cabernet Sauvignon wines originating from countries with similar agro-climatic characteristics in the Balkan region (Croatia, Bosnia and Herzegovina, Montenegro, Serbia, Macedonia and Bulgaria), indicating similar profiles of phenolic compounds.

\section{CONCLUSIONS}

In our work, the HPLC method with spectrofluorometric detection was used for an analysis of gentisic and salicylic acids in a wide range of wines, assuring acceptable detection and quantification limits despite the direct injection of wine samples. The proposed procedure simplified the preparation of a sample prior to HPLC analysis, and achieved satisfactory separation of chromatographic peaks of both phenolic acids and LOD and LOQ values. 
In this work, the content of gentisic and salicylic acids, as well as total phenolic compounds in wines produced from several grape varieties, was analysed. Furthermore, the inhibition of $\mathrm{AChE}$ and $\mathrm{BChE}$ by wines was determined for the first time. The solutions of gentisic and salicylic acids were prepared at concentrations similar to those detected for both phenolic acids in red wines; it was observed that both phenolic acids are weak cholinesterase inhibitors at the maximal concentrations found in wines studied in our work. However, red wines produced from different grape varieties, as a whole, displayed significant inhibition of $\mathrm{AChE}$ and BChE.

Wines made from different grape cultivars examined in this work could be divided into three broad groups based on chemometric analysis. The highest salicylic acid content and AChE inhibitory activity, and lower BChE inhibitory activity, were shown by Cabernet Sauvignon and Syrah wines. Garnacha tinta, Merlot, Montepulciano and Negroamaro wines showed a medium content of salicylic acid, and highest or medium BChE inhibitory activity. The lowest salicylic acid content and low AChE inhibitory activity was exhibited by the Pinot noir, Tempranillo, Regent and Rondo cultivars. Of the Polish varieties studied, Regent wines had the highest content of gentisic acid, medium TPC and BChE inhibitory activity, and low salicylic acid content and AChE inhibitory activity. Rondo wines were characterised by a medium content of gentisic acid, TPC and BChE inhibitory activity, low AChE inhibitory activity and the lowest content of salicylic acid.

To the best of our knowledge, no previous studies have been published on red wine compounds as cholinesterase inhibitors. These results are therefore important in helping to elucidate natural cholinesterase inhibitors found in foods, and indicate that the inclusion of red wine in a balanced diet may help prevent Alzheimer's disease.

\section{LITERATURE CITED}

Anand, A., Patience, A.A., Sharma, N. \& Khurana, N., 2017. The present and future of pharmacotherapy of Alzheimer's disease: A comprehensive review. Eur. J. Pharmacol. 815, 364-375.

Barnaba, C., Dellacassa, E., Nicolini, G., Nardin, T., Malacarne, M. \& Larcher R., 2016. Free and glycosylated simple phenol profiling in Apulian Italian wines. Food Chem. 206, 260-266.

Borowiec, K., Szwajgier, D., Targoński, Z., Demchuk, O.M., Cybulska, J., Czernecki, T. \& Malik, A., 2014. Cholinesterase inhibitors isolated from bilberry fruit. J. Funct. Food 11, 313-321.

Clarke, G., Ting, K.N., Wiart, C. \& Fry, J., 2013. High correlation of 2,2-diphenyl-1-picrylhydrazyl (DPPH) radical scavenging, ferric reducing activity potential and total phenolics content indicates redundancy in use of all three assays to screen for antioxidant activity of extracts of plants from the Malaysian rainforest. Antioxidants 2(1), 1-10.

Cotea, V.V., Luchian, C.E., Bilba, N. \& Niculaua, M., 2012. Mesoporous silica SBA-15, a new adsorbent for bioactive polyphenols from red wine. Anal. Chim. Acta 732, 180-185.

Del Alamo Sanza, M., Dominguez, I.N., Cárcel Cárcel, L.M. \& Navas Gracia, L., 2004. Analysis for low molecular weight phenolic compounds in a red wine aged in oak chips. Anal. Chim. Acta 513, 229-237.
Ellman, G.L., Courtney, K.D., Andres, V. \& Featherstone, R.M., 1961. A new and rapid colorimetric determination of acetylcholinesterase activity. Biochem. Pharmacol. 7, 88-95.

Erdem, S.A., Senol, F.S., Budakoglu, E., Orhan, I.E. \& Sener, B., 2016. Exploring in vitro neurobiological effects and high-pressure liquid chromatography-assisted quantitation of chlorogenic acid in 18 Turkish coffee brands. J. Food Drug Anal. 24, 112-120.

Fanzone, M., Peña-Neira, A., Gil, M., Jofré, V., Assof, M. \& Zamora, F., 2012. Impact of phenolic and polysaccharidic composition on commercial value of Argentinean Malbec and Cabernet Sauvignon wines. Food Res. Int $45,402-414$.

Garmón-Lobato, S., López-Márquez, D.M., Sánchez-Fernández, C., Berrueta, L.A., Gallo, B., Vicente, F. \& Arranz, A., (2004). Study of native fluorescence of phenolic compounds for monitoring HPLC separations and their characterization [Online]: http://www.ehu.eus/XV_SEQA/Resumenes/ Posters_2/C2-124.pdf [accessed 22 January 2018].

Ginjom, I.R., D’Arcy, B.R., Caffin, N.A. \& Gidley, M.J., 2010. Phenolic contents and antioxidant activities of major Australian red wines throughout the winemaking process. J. Agric. Food Chem. 58, 10133-10142.

Gironés-Vilaplana, A., Valentão, P., Andrade, P.B., Ferreres, F., Moreno, D.A. \& García-Viguera, C., 2012. Phytochemical profile of a blend of black chokeberry and lemon juice with cholinesterase inhibitory effect and antioxidant potential. Food Chem. 134, 2090-2096.

Gonçalves, J., Silva, C.L., Castilho, P.C. \& Câmara, J.S., 2013. An attractive, sensitive and high-throughput strategy based on microextraction by packed sorbent followed by UHPLC-PDA analysis for quantification of hydroxybenzoic and hydroxycinnamic acids in wines. Microchem. J. 106, 129-138.

Ivanova-Petropulos, V., Ricci, A., Nedelkovski, D., Dimovska, V., Parpinello, G.P., \& Versari, A., 2015. Targeted analysis of bioactive phenolic compounds and antioxidant activity of Macedonian red wines. Food Chem. $171,412-420$.

Jin, X.D., Wu, X. \& Liu, X., 2017. Phenolic characteristics and antioxidant activity of Merlot and Cabernet Sauvignon wines increase with vineyard altitude in a high-altitude region. S. Afr. J. Enol. Vitic. 38, 132-143.

Kapusta, I., Cebulak, T. \& Oszmiański, J., 2018. Characterization of Polish wines produced from the interspecific hybrid grapes grown in south-east Poland. Eur. Food Res. Technol. 244, 441-455.

Kim, J.W., Lee, D.Y., Lee, B.C., Jung, M.H., Kim, H., Choi, Y.S. \& Choi, I.G., 2012. Alcohol and cognition in the elderly: A review. Psychiatry Investig. 9, 8-16.

Kumar, K., Kumar, A., Keegan, R.M. \& Deshmukh, R., 2018. Recent advances in the neurobiology and neuropharmacology of Alzheimer's disease. Biomed. Pharmacother. 98, 297-307.

Ma, T.-T., Sun, X.-Y., Gao, G.-T., Wang, X.-Y., Liu, X.-Y., Du, G.-R. \& Zhan, J.-C., 2014. Phenolic characterisation and antioxidant capacity of young wines made from different grape varieties grown in Helanshan Donglu wine zone (China). S. Afr. J. Enol. Vitic, 35, 321-331.

Nikfardjam, M.P., Patz, C.D., Kauer, R., Schultz, H. \& Dietrich, H., 1999. A modified HPLC method to detect salicylic acid in must and wine after its application in the field to induce fungus resistance. Vitis $38,179-182$.

Nooyens, A.C., Bueno-de-Mesquita, H.B., Van Gelder, B.M., Van Boxtel, M.P. \& Verschuren, W.M., 2014. Consumption of alcoholic beverages and cognitive decline at middle age: The Doetinchem Cohort Study. Brit. J. Nutr. 111, 715-723

Nwanna, E.E., Oyeleye, S.I., Ogunsuyi, O.B., Oboh, G., Boligon, A.A. \& Athayde, M.L., 2016. In vitro neuroprotective properties of some commonly consumed green leafy vegetables in Southern Nigeria. NFS Journal 2, 1924. 
Okello, E.J., Leylabi, R. \& McDougall, G.J., 2012. Inhibition of acetylcholinesterase by green and white tea and their simulated intestinal metabolites. Food Funct. 3, 651-661.

Pereira, V., Câmara, J.S., Cacho, J. \& Marques, J.C., 2010. HPLC- DAD methodology for the quantification of organic acids, furans and polyphenols by direct injection of wine samples. J. Sep. Sci. 33, 1204-1215.

Raczkowska, J., Mielcarz, G., Howard, A. \& Raczkowski, M., 2011. UPLC and spectrophotometric analysis of polyphenols in wines available in the Polish market. Int. J. Food Prop. 14, 514-522.

Radovanovic, A., Jovancicevic, B., Arsic, B., Radovanovic, B. \& Bucarica L., 2016. Application of non-supervised pattern recognition techniques to classify Cabernet Sauvignon wines from the Balkan region based on individual phenolic compounds. J. Food Compos. Anal. 49, 42-48.

Ren, M., Wang, X., Du, G., Tian, C., Zhang, J., Song, X. \& Zhu, D., 2017. Influence of different phenolic fractions on red wine astringency based on polyphenol/protein binding. S. Afr. J. Enol. Vitic. 38, 118-124.

Rhee, I.K., Van Rijn, R.M. \& Verpoorte, R., 2003. Qualitative determination of false- positive effects in the acetylcholinesterase assay using thin layer chromatography. Phytochem. Anal. 14, 127-131.

Romesburg, H.C., 2004 (reprint of 1984 edition, with minor revisions). Cluster analysis for researchers. Lulu.com, Morrisville, NC.

Sen, I. \& Tokatli, F., 2014. Authenticity of wines made with economically important grape varieties grown in Anatolia by their phenolic profiles. Food Control 46, 446-454

Socha, R., Gałkowska, D., Robak, J., Fortuna, T. \& Buksa, K., 2015. Characterization of Polish wines produced from the multispecies hybrid and Vitis vinifera L. grapes. Int. J. Food Prop. 18, 699-713.

Stockley, C.S., 2015. Wine consumption, cognitive function and dementias - A relationship? Nutrition and Aging 3, 125-137.

Szwajgier, D. \& Borowiec, K., 2012a. Phenolic acids from malt are efficient acetylcholinesterase and butyrylcholinesterase inhibitors. J. Inst. Brew. 118, 40-48.
Szwajgier, D. \& Borowiec, K., 2012b. Screening for cholinesterase inhibitors in selected fruits and vegetables. EJPAU 15(2), \#06.

Szwajgier, D., 2013a. Anticholinesterase activity of phenolic acids and their derivatives. Z. Naturforsch. C 68, 125-132.

Szwajgier, D., 2013b. Inhibition of cholinesterases by phenolic acids detected in beer: A dose-response model approach. Afr. J. Biotechnol. 12, 1675-1681.

Tomaz, I. \& Maslov, L., 2016. Simultaneous determination of phenolic compounds in different matrices using phenyl-hexyl stationary phase. Food Anal. Method. 9, 401-410.

Van Leeuw, R., Kevers, C., Pincemail, J., Defraigne, J.O. \& Dommes, J., 2014. Antioxidant capacity and phenolic composition of red wines from various grape varieties: Specificity of Pinot noir. J. Food Compos. Anal. 36, $40-50$.

Vitrac, X., Monti, J.P., Vercauteren, J., Deffieux, G. \& Mérillon, J.M., 2002. Direct liquid chromatographic analysis of resveratrol derivatives and flavanonols in wines with absorbance and fluorescence detection. Anal. Chim. Acta 458, 103-110.

Wang, J., Ho, L., Zhao, Z., Seror, I., Humala, N., Dickstein, D.L., Thiyagarajan, M., Percival, S.S., Talcott, S.T. \& Pasinetti, G.M., 2006. Moderate consumption of Cabernet Sauvignon attenuates $A \beta$ neuropathology in a mouse model of Alzheimer's disease. FASEB J. 20, 2313-2320.

Wang, J., Santa-Maria, I., Ho, L., Ksiezak-Reding, H., Ono, K., Teplow, D.B. \& Pasinetti, G.M., 2010. Grape derived polyphenols attenuate tau neuropathology in a mouse model of Alzheimer's disease. J. Alzheimers Dis. 22, 653-661.

Wojdyło, A., Samoticha, J., Nowicka, P. \& Chmielewska, J., 2018. Characterisation of (poly)phenolic constituents of two interspecific red hybrids of Rondo and Regent (Vitis vinifera) by LC-PDA-ESI-MS QTof. Food Chem. 239, 94-101.

Xi, Z.M., Zhang, Z.W., Cheng, Y.F. \& Hua, L.I., 2010. The effect of vineyard cover crop on main monomeric phenols of grape berry and wine in Vitis vinifera L. cv. Cabernet Sauvignon. Agric. Sci. China, 9, 440-448. 